Article

\title{
Experimental Runaway Electron Current Estimation in COMPASS Tokamak
}

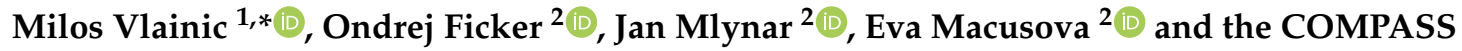 \\ Tokamak Team \\ 1 Institute of Physics, University of Belgrade, Pregrevica 118, P.O. Box 68, 11080 Belgrade, Serbia \\ 2 Institute of Plasma Physics, Czech Academy of Sciences-Institute of Plasma Physics, Za Slovankou 1782/3, \\ 18200 Prague 8, Czech Republic; ficker@ipp.cas.cz (O.F.); mlynar@ipp.cas.cz (J.M.); \\ macusova@ipp.cas.cz (E.M.) \\ * Correspondence: milos.vlainic@ipb.ac.rs
}

Received: 30 November 2018; Accepted: 13 January 2019; Published: 16 January 2019

check for updates

\begin{abstract}
Runaway electrons present a potential threat to the safe operation of future nuclear fusion large facilities based on the tokamak principle (e.g., ITER). The article presents an implementation of runaway electron current estimations at COMPASS tokamak. The method uses a theoretical method developed by Fujita et al., with the difference in using experimental measurements from EFIT and Thomson scattering. The procedure was explained on the COMPASS discharge number 7298, which has a significant runaway electron population. Here, it was found that at least $4 \mathrm{kA}$ of the plasma current is driven by the runaway electrons. Next, the method aws used on the set of plasma discharges with the variable electron plasma density. The difference in the plasma current was explained by runaway electrons, and their current was estimated using the aforementioned method. The experimental results are compared with the theory and simulation. The comparison presented some disagreements, showing the possible direction for the code development. Additional application on runaway electron energy limit is also addressed.
\end{abstract}

Keywords: runway electron; plasma current; fusion plasma; tokamak

\section{Introduction}

In the last decade, nuclear fusion started to shift from science to industry, where the tokamak-based ITER is playing the leading role. Runaway electrons (REs) present a potential threat to the safe operation of the future nuclear fusion power plants based on the tokamak principle. Namely, in the latest issue of ITER Physics Basis [1], REs are considered as the second-highest priority for the ITER disruption mitigation.

COMPASS tokamak [2] is a suitable and low-risk test bed for studying RE disruption physics and RE model benchmarking. However, one of the common missing RE parameters for comparison of experiment and simulation is RE current or RE density. This article addresses this issue for certain cases.

The basic idea behind the used model was reported on ORMAK tokamak by Fujita [3]. In contrast to Fujita's full-theoretical approach, we implemented experimental data from EFIT [4] and Thomson Scattering [5] to the calculation. The obtained results are in the acceptable range and their application on density scans and RE localization is presented.

The article starts with a description of an experimental observation of the particular discharge. Theoretical models and detailed description of the settings used in the models for comparison with the experiments are then reported. An estimation of the RE current $I_{R E}$ is the first experimental analysis. Subsequently, the influence of the REs on the current ramp-up phase of the discharge is evaluated. Following that, a principle on how to localize RE beam is done in the next section, using the knowledge 
of the RE beam current and its maximum energy. Finally, discussions and conclusions are given in the last section with an outlook towards expected future works.

\section{Experimental Setup: COMPASS Tokamak}

The COMPASS tokamak is a compact experimental fusion facility with a major radius $R_{0}=0.56 \mathrm{~m}$ and a minor radius $a_{p}=0.23 \mathrm{~m}$. The toroidal magnetic field $B_{\text {tor }}$ is in the $0.9-1.6 \mathrm{~T}$ range (typically set to $1.15 \mathrm{~T}$ ), coming from 16 toroidal field coils. The plasma current $I_{p}$ can reach up to $400 \mathrm{kA}$ using an air-cored transformer. The range for the electron densities is flexible and is typically in the $10^{19}-10^{20} \mathrm{~m}^{-3}$ domain. Plasma shaping varies from circular and elliptical to single-null D-shaped ITER-like plasmas. When circular, the plasma is limited by a carbon HFS wall. The regular pulse length is $\sim 0.4 \mathrm{~s}$, although low current circular discharges with RE can last almost $1 \mathrm{~s}[6,7]$.

The Thomson Scattering diagnostic system in COMPASS uses two Nd:YAG lasers of wavelength $1064 \mathrm{~nm}$ with an energy of $1.5 \mathrm{~J}$ for each laser. The whole system is oriented vertically and the scattered light is recorded in the radial direction. Each laser has $30 \mathrm{~Hz}$ repetition rate, which offers a $\sim 16.7 \mathrm{~ms}$ time resolution if the two lasers are operated equidistantly.

The EFIT reconstruction provides relevant quantities on a 2D mesh cross-section of the poloidal plasma plane solving the Grad-Shafranov equation for plasma MHD equilibrium, constrained by the magnetic diagnostics at the COMPASS tokamak (current measured in plasma and field coils, pick-up coils). Additionally, in the analysis addressed here, a toroidal loop voltage in plasma $V_{\text {plasma }}$ for computing a maximum RE energy $W_{\max }$ is calculated using METIS [8], a Multi-Element Tokamak-oriented Integrated Simulator.

\section{Experiment: High RE Current Discharge}

The very first magnetic observations of high RE current in the COMPASS tokamak are reported in Ref. [9] for discharge \#7298. The discharge had circular-shaped limited plasma, with toroidal magnetic field $B_{t o r}=1.15 \mathrm{~T}$ and plasma current $I_{p}=120 \mathrm{kA}$. The plasma density $n_{e}$ was lower than $1.5 \times 10^{19} \mathrm{~m}^{-3}$ in the plasma core as measured by the Thomson Scattering system.

The COMPASS tokamak is well-supplied with the general plasma measurements, which provided us with the following list of observations in the discharge:

- $\quad$ Loop voltage measured plasma voltage $V_{\text {plasma }}$ bellow $1 \mathrm{~V}$, while the typical range is $1.5-2 \mathrm{~V}$;

- Electron temperature from Thomson Scattering was 1 order of magnitude colder than ordinary tokamak plasma;

- Hard X-ray scintillators observed a significant amount of RE losses;

- Poloidal internal pick-up coils detected inner structure that is moving outwards;

- Vertical field for feedback of horizontal plasma position was increasing during the discharge, even though plasma current was constant;

- EFIT reconstruction of poloidal cross-section depicted plasma shrinkage towards inner side, due to the increasing magnetic pressure coming from the above-mentioned vertical field;

- $\quad$ Plasma pressure in terms of its ratio to the poloidal magnetic field pressure (i.e., poloidal beta $\left.\beta_{\text {pol }}\right)$, estimated from EFIT showed unrealistic large values.

All aforementioned information provides us with enough evidence to conclude that RE beam co-existed with the bulk plasma. For more detailed discussion on the measurements, the interested reader is referred to Section 5.1 in Ref. [10].

\section{Method: RE Current Calculation}

The overestimate of the $\beta_{p o l}$ is exploited here for the estimate of the RE current $I_{R E}$. The analysis is based on work done by Fujita et al. [3]. Even though the basic principle used here is the same as Fujita's, the final approach to the calculation is different. While the original paper estimates all necessary parameters theoretically, we use direct measurements from the Thomson scattering and 
calculations from EFIT reconstruction. In this section, we only address our changes to the method. The main idea behind the Fujita's model is an estimate of RE pressure $P_{R E}$ from the difference between total plasma pressure $p_{\text {tot }}$ (coming from EFIT $\beta_{\text {pol }}$ )

$$
\left\langle p_{t o t}\right\rangle_{V}=\frac{\beta_{p o l} B_{p o l}^{2}}{2 \mu_{0}} \approx 5 \times 10^{-8} \frac{\beta_{p o l}}{\pi}\left(\frac{I_{p}}{a_{p}}\right)^{2}
$$

and pure bulk plasma pressure $p_{p l}$ (coming from the Thomson Scattering measured radial $T_{e}$ profile)

$$
\left\langle p_{p l}\right\rangle_{V}=\frac{2}{a_{p}^{2}} \int_{0}^{a_{p}} p_{p l}(r) r \mathrm{~d} r
$$

where $r$ is the radial position along the minor radius $a_{p}$. Examples of $\left\langle p_{t o t}\right\rangle_{V}$ and $\left\langle p_{p l}\right\rangle_{V}$ are presented in Figure 1.

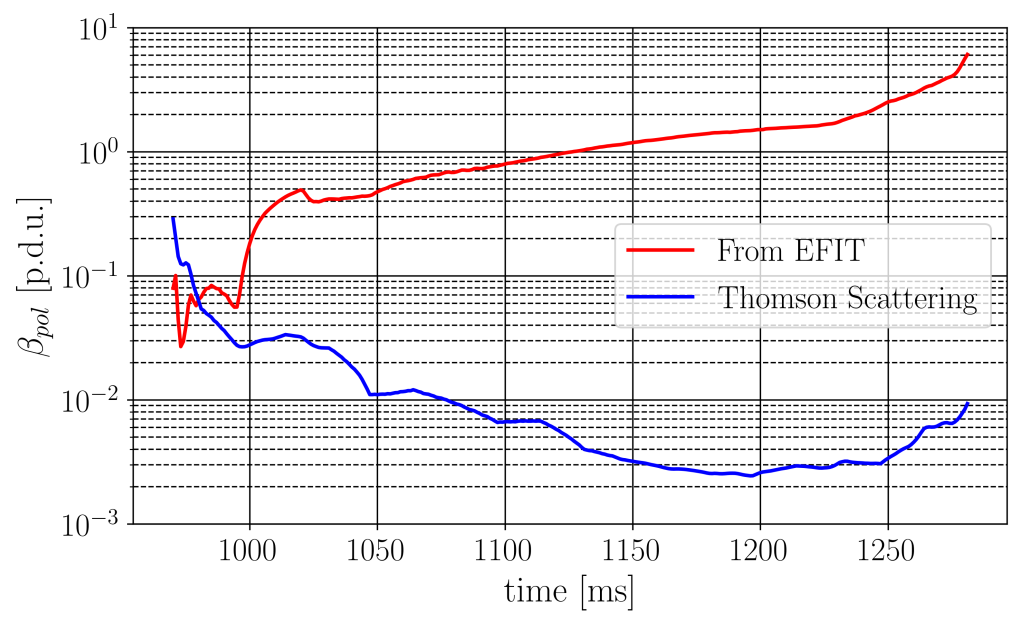

Figure 1. Poloidal beta $\beta_{\text {pol }}$ estimated by EFIT (red) and from Thomson Scattering (blue) for discharge \#7298.

The $P_{R E}=\left\langle p_{t o t}\right\rangle_{V}-\left\langle p_{p l}\right\rangle_{V}$ is calculated as an average from obtained $P_{R E}$ values corresponding to results when following conditions $\left\langle p_{t o t}\right\rangle_{V}>1.4\left\langle p_{p l}\right\rangle_{V}$ and $\left\langle p_{t o t}\right\rangle_{V}>2\left\langle p_{p l}\right\rangle_{V}$ are satisfied simultaneously, to reduce the propagation of error given by this approach. The first condition specifies the accuracy of Thomson Scattering measurements and thereby defines the minimal threshold necessary to distinguish between $\left\langle p_{t o t}\right\rangle_{V}$ and $\left\langle p_{p l}\right\rangle_{V}$ and eliminates the error of Thomson Scattering measurements. The second condition is always valid in the case of non-negligible RE pressure and it is artificially taken as a conservative limit.

Furthermore, $P_{R E}$ is connected to the RE density

$$
P_{R E}=\left\langle p_{R E}\right\rangle=\frac{1}{2} m_{e}\left\langle n_{R E}\right\rangle\left\langle\gamma v_{\|}^{2}\right\rangle+\frac{1}{4} m_{e}\left\langle n_{R E}\right\rangle\left\langle\gamma v_{\perp}^{2}\right\rangle
$$

where \langle\rangle represents average in both spatial and momentum coordinates, $\gamma$ is relativistic Lorentz factor, and $v_{\|}$and $v_{\perp}$ denote the RE velocity in the parallel and perpendicular direction to the magnetic field $\vec{B}$, respectively. By estimating the RE energy, one can estimate the RE density $\left\langle n_{R E}\right\rangle$, from which RE current $I_{R E}$ can be approximately derived using the following expression:

$$
I_{R E}=e c\left\langle n_{R E}\right\rangle\langle\beta\rangle A_{R E}
$$

where $A_{R E}$ is the area of the RE beam cross-section and $\beta$ is normalized relativistic velocity. 


\subsection{RE Energy Calculation}

The model for maximum obtainable RE energy $W_{\max }$ is based on Ref. [11], where radiation losses are subtracted from the acceleration. It is a $0 \mathrm{D}$ model, as there is no dimensional dependency, but only time evolution. Particularly, the lost power by synchrotron radiation $P_{\text {synch }}$ and bremsstrahlung radiation $P_{\text {brems }}$ are subtracted from the power gained by the RE thanks to the electric field $P_{E}$

$$
\frac{\mathrm{d} W_{\max }}{\mathrm{d} t}=P_{E}-P_{\text {synch }}
$$

to obtain the time dependence of $W_{\max }$. The power gain by the toroidal electric field $E_{\text {tor }}$ is simply calculated as

$$
P_{E}=e c E_{t o r}=\frac{e c V_{\text {plasma }}}{2 \pi R_{p}}
$$

with $R_{p}$ standing for the plasma major radius. The synchrotron power loss

$$
P_{\text {synch }}=\frac{2 r_{e} m_{e} c^{3} \beta^{4} \gamma^{4}}{3 R_{c}^{2}}
$$

where $r_{e}$ and $m_{e}$ are the classical electron radius and mass, respectively. The curvature radius $R_{c}$ is calculated using Equation (9) from Ref. [12]:

$$
R_{c}=\frac{R_{p}}{1+\eta^{2}+2 \eta \times 2 / \pi}
$$

where $2 / \pi$ at the end of the denominator comes from the average taken over the gyro-motion angle, and where $\eta=\left\langle v_{\perp}\right\rangle / v_{d r}$ and $v_{d r}$ is the drift velocity

$$
v_{d r}=\frac{\langle\gamma\rangle\left\langle v_{\|}^{2}\right\rangle}{\Omega R_{p}}
$$

with $\Omega$ being the fundamental gyro-frequency. Finally, the angle brackets are making the whole calculation dependent on the RE distribution function (REDF).

\subsection{RE Distribution Functions}

With mentioning REDF, we need to address its influence on the $I_{R E}$ calculation. The REDFs used by Fujita et al. and utilized here are monoenergetic $f_{\text {mono }}$, uniform $f_{\text {uni }}$, and linear $f_{\text {lin }}$. The definitions of the first three distributions as functions of RE energy $w$ are as follows:

$$
\begin{aligned}
f_{\text {mono }}(w) & =\delta\left(w-W_{\max }\right), \\
f_{\text {uni }}(w) & =\frac{1}{W_{\max }}, \\
f_{\text {lin }}(w) & =\frac{2}{W_{\max }}\left(1-\frac{w}{W_{\max }}\right),
\end{aligned}
$$

with $\delta()$ being the Dirac delta function. The coefficients come from setting the REDF maximum value to 0 at $W_{\max }$ and then normalizing the REDF to 1.

Furthermore, the exponential REDF

$$
f_{\exp }(w ; \epsilon)=\epsilon e^{-\epsilon w}
$$


is introduced to present a distribution with a steep decrease that could be important if the avalanche mechanism were to dominate. Additionally, two more REDFs are considered here: skewed Gaussian $f_{s G}[13]$

$$
\begin{gathered}
f_{s G}(w ; l, \zeta, \alpha)=\frac{2}{\zeta} f_{G p d f}(s) f_{G c d f}(s \alpha) \\
f_{G p d f}(s)=\frac{1}{\sqrt{2 \pi}} e^{-s^{2} / 2} \\
f_{G c d f}(s \alpha)=\frac{1}{2}\left[1+\operatorname{erf}\left(\frac{s \alpha}{\sqrt{2}}\right)\right]
\end{gathered}
$$

with $s=(w-l) / \zeta$ and Maxwell-Jüttner $f_{M J}[14]$

$$
f_{M J}(w ; \mathcal{W})=\frac{\beta \gamma^{2}}{\mathcal{W} K_{2}(1 / \mathcal{W})} e^{-\gamma / \mathcal{W}}
$$

distributions. The (negatively) skewed Gaussian represents a more realistic case of $f_{\text {mono, }}$ as it is not a delta-function. In other words, the negatively skewed Gaussian represents REDF where almost all RE tend towards $W_{\max }$ but they never reach it. The Maxwell-Jüttner distribution function $f_{M J}$ is selected as one of the most commonly relativistic distribution functions used in the literature. As all three latter functions have asymptotic behavior towards zero and/or $W_{\text {max }}$, normalization has to be different than for the former three. Their parameters are set in such a way that asymptotic edge is smaller than $10^{-5}$, while $f_{s G}$ maximum is located just over $w=0.95 W_{\max }$.

\subsection{Pitch Angle}

Equation (3) indicates that the pressure separation through the velocities $v_{\|}$and $v_{\perp}$ implies knowing the pitch angle $\theta$. Fujita et al. omitted the $\left\langle\gamma v_{\perp}^{2}\right\rangle$ term and approximated the other one $\left\langle\gamma v_{\|}^{2}\right\rangle$ by $c^{2}\left\langle\gamma \beta^{2}\right\rangle$. However, we consider the $\theta$ influence to be consistent with the estimation of $W_{\max }$ reported in Section 4.1, where $\theta$ plays a role through the parameter $\eta \propto\left\langle\beta_{\perp}\right\rangle /\left(\langle\gamma\rangle\left\langle\beta_{\|}^{2}\right\rangle\right)$.

Table 1 shows the influence of different REDFs on essential parameters for $I_{R E}$ calculation. Firstly, it is important to state that averages presented in Table 1 are done for energy span from 0 to $W_{\max }$ using the mean of the variable, i.e., neglecting the RE limit at lower energies. The error is negligible due to the high $W_{\text {max }}$ reached in the COMPASS discharges. The average energy $\langle w\rangle$ is calculated for illustration, while $\langle\beta\rangle$ can be seen in Equation (4).

Table 1. Comparison of parameters (RE energy $w$, relativistic $\beta$, the term from Equation (3) $\left\langle\beta^{2} \gamma\right\rangle=$ $\left\langle\beta_{\|}^{2} \gamma\right\rangle+0.5\left\langle\beta_{\perp}^{2} \gamma\right\rangle$ and drift term $\eta$ for $W_{\max }$ estimation) averaged over $w$ for different REDFs.

\begin{tabular}{rcccccc}
\hline \multirow{2}{*}{ REDF } & \multirow{2}{*}{$\boldsymbol{w}\rangle[\mathbf{M e V}]$} & $\langle\boldsymbol{\beta}\rangle$ & \multicolumn{2}{c}{$\left\langle\boldsymbol{\beta}^{\mathbf{2}} \gamma\right\rangle$} & \multicolumn{2}{c}{$\langle\boldsymbol{\eta}\rangle$} \\
\cline { 4 - 7 } & & & $\boldsymbol{\theta}=\mathbf{0 . 0}$ & $\boldsymbol{\theta}=\mathbf{0 . 3}$ & $\boldsymbol{\theta}=\mathbf{0 . 1}$ & $\boldsymbol{\theta}=\mathbf{0 . 3}$ \\
\hline$f_{\text {mono }}$ & 10.00 & 0.9988 & 20.5 & 19.6 & 4.91 & 15.8 \\
$f_{\text {uni }}$ & 5.00 & 0.9721 & 10.6 & 10.2 & 9.55 & 30.7 \\
$f_{\text {lin }}$ & 3.33 & 0.9497 & 7.30 & 6.98 & 13.9 & 44.7 \\
$f_{\text {exp }}$ & 0.86 & 0.8141 & 2.18 & 2.09 & 43.8 & 140 \\
$f_{\text {sG }}$ & 8.02 & 0.9980 & 16.6 & 15.9 & 6.05 & 19.4 \\
$f_{M J}$ & 1.58 & 0.9301 & 3.78 & 3.62 & 26.21 & 84.2 \\
\hline
\end{tabular}

From Equation (4), one would expect that the exponential REDF has the smallest $I_{R E}$ for the same $P_{R E}$, as it has the far smallest $\langle\beta\rangle$. However, due to the term $\left\langle\beta_{\|}^{2} \gamma\right\rangle+0.5\left\langle\beta_{\perp}^{2} \gamma\right\rangle$ in Equation (3) used for the $\left\langle n_{R E}\right\rangle$ estimation (by knowing $P_{R E}$ in Equation (3)), the result is opposite. Namely, difference in $\beta^{2} \gamma$ from Table 1 of an order of magnitude creates much larger difference in estimated $n_{R E}$ between 
REDFs than $\langle\beta\rangle$ does in Equation (4). Therefore, even though the exponential has by far the smallest $\langle\beta\rangle$, it has the largest $I_{R E}$.

Furthermore, even though two extreme values are taken for $\theta$ to investigate maximum possible effect from the corresponding variations, the difference due to $\left\langle\beta^{2} \gamma\right\rangle$ is smaller than a few percent for every REDF. Furthermore, the aforementioned $\eta$ is calculated for $\theta=\{0.1,0.3\}$ rad, as it is zero at $\theta=0 \mathrm{rad}$. Here, a significant influence of the pitch angle on the $\eta$ is observed. Henceforth, $\theta$ is implemented in $\eta$ that is relevant for $W_{\max }$ calculation. Another expected dependence that can be noted from Table 1 is that the most similar REDF to the monoenergetic one is the skewed Gaussian $f_{s G}$ REDF, as their parameters are close to each other.

\section{Result: COMPASS Discharge 7298}

The pitch angle $\theta$ is taken to be $0.2 \mathrm{rad}$ as a mean value found in Ref. [15]. The RE minor radius $a_{R E}$ for the RE area $A_{R E}$ calculation is assumed to be constant and equal to $5 \mathrm{~cm}$. The METIS (with the effective ion charge $Z_{e f f}=2.5$ ) data for the electric field and plasma pressure $\left\langle p_{p l}\right\rangle_{V}$ from Thomson scattering are interpolated and extrapolated to the EFIT time scale. As EFIT measures before the first and after the last Thomson scattering measurement, the first and the last point of the extrapolated $\left\langle p_{p l}\right\rangle_{V}$ are approximated to be $95 \%$ (arbitrarily taken) of the first and the last Thomson scattering measurement, respectively.

The main result is presented in Figure 2. The estimate of the RE current $I_{R E}$ for different RE distribution functions are presented in Figure $2 \mathrm{c}$ and their maximum values are listed in Table 2. The plasma current $I_{p}$ is plotted in Figure $2 \mathrm{~b}$ for comparison purposes. In the same figure, Shielded HXR and Standard HXR are also plotted.
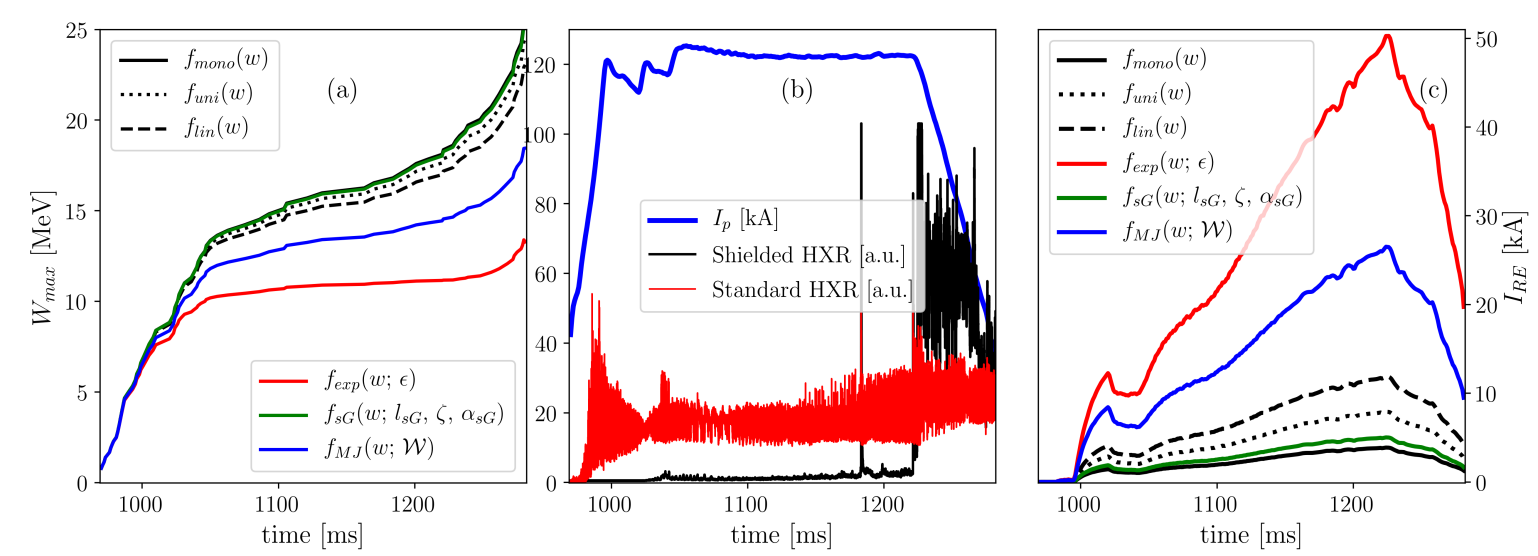

Figure 2. (a) The maximum kinetic energy $W_{\max }$ of discharge $\# 7298$ obtained for the different $R E$ distribution functions: mono-energetic $f_{\text {mono }}$ (black solid), uniform $f_{\text {uni }}$ (black dotted), linear $f_{\text {lin }}$ (black dashed), exponential $f_{\text {exp }}$ (red), skewed Gaussian $f_{s G}$ (green) and Maxwell-Jüttner $f_{M J}$ (blue). (b) Time traces of the plasma current $I_{p}$ (blue), Shielded HXR (black) and Standard HXR (red). (c) Estimated RE current $I_{R E}$ corresponding to each RE distribution function with the same labeling as on (a).

The comparison between the $W_{\max }$ estimate coming from different $\mathrm{RE}$ distribution functions described in Section 4.2 is shown in Figure 2a and their maximum values are tabulated in Table 2.

In Figure $2 a$, one can see that only $W_{\max }$ from the exponential $f_{\exp }$ and the Maxwell-Jüttner $f_{M J}$ distribution function are significantly different, as could be expected from the $\langle\eta\rangle$ values in Table 1.

Even though $\beta_{\text {pol }}$ from EFIT increases significantly during the ramp-down phase of the discharge, a relatively strong decrease in RE current $I_{R E}$ can be seen in Figure 2c. This is a consequence of the current drop in the total plasma pressure $\left\langle p_{t o t}\right\rangle_{V}$ calculation from EFIT in Equation (1). It is interesting that the $I_{R E}$ drop coincides with the RE losses seen from Shielded HXR signal, even though RE losses are not implemented in the calculation. Henceforth, the $I_{R E}$ decrease can be seen as numerical artifact. 
However, physical interpretation could be an additional RE energy limit due to the RE beam drift, which is reported in Section 7. Next, notice that $I_{R E}$ values span over two orders of magnitude-from a few $\mathrm{kA}$ to tens of $\mathrm{kA}$.

Even though $f_{\text {mono }}$ is the most unrealistic of all the used RE distribution functions, it gives minimum $I_{R E}$ as $\beta, \gamma$ and $\eta$ have extreme values. Therefore, we conclude that at least a few $\mathrm{kA}$ of current should definitely be driven by the REs in the COMPASS discharge \#7298. However, it is not possible to determine which REDF gives the most accurate RE current $I_{R E}$ estimate from Figure 2.

The above experimental results are compared here with the Kruskal-Bernstein theory [16] and NORSE simulation [17] in Figure 3. Kruskal-Bernstein theory is the analytical solution for growth rate of the Dreicer mechanism of RE generation, whose last shape was derived by Connor and Hastie [16]. The Kruskal-Bernstein equation is a strong function of the $E_{t o r}$. NORSE calculateS only Dreicer mechanism of RE generation and $n_{e}, T_{e}$ and $E_{t o r}$ are taken as time-varying parameters, while $Z_{e f f}$ and $B_{t o r}$ are constant. Finally, the $n_{R E}$ is computed by multiplying the total electron density from measurements $n_{e, c}$ with the RE fraction calculated by NORSE.

When applied to the COMPASS discharge \#7298, the Kruskal-Bernstein theory predicts RE density $n_{R E}$ larger by 1-3 orders of magnitude than the total $n_{e}$. This is a non-physical result of course and we consider this theory not relevant for this particular case. Furthermore, NORSE reaches slide-away regime (i.e., when all electrons runaway) at the very beginning of the discharge. Such early slide-away regime is not supported by the experimental results.

Table 2. The maxima of $W_{\max }$ and $I_{R E}$ over the time domain of the whole discharge, plotted in Figure 2.

\begin{tabular}{ccc}
\hline REDF & $\max \left(\boldsymbol{W}_{\text {max }}\right)[\mathbf{M e V}]$ & $\max \left(\boldsymbol{I}_{\boldsymbol{R E}}\right)[\mathbf{k A}]$ \\
\hline$f_{\text {mono }}$ & 25.61 & 3.9 \\
$f_{\text {uni }}$ & 24.49 & 7.9 \\
$f_{\text {lin }}$ & 23.05 & 11.9 \\
$f_{\text {exp }}$ & 13.39 & 50.3 \\
$f_{\text {sG }}$ & 25.37 & 5.0 \\
$f_{M J}$ & 18.54 & 26.5 \\
\hline
\end{tabular}

The possible reasons for the overestimating results from Kruskal-Bernstein theory and NORSE simulation could result from overestimating the electric field $E_{t o r}$ by METIS or from too high sensitivity of the theories on the $E_{t o r}$ parameter. Moreover, RE energy calculation used here is also simplistic and EFIT results could be misleading in the presence of the RE.

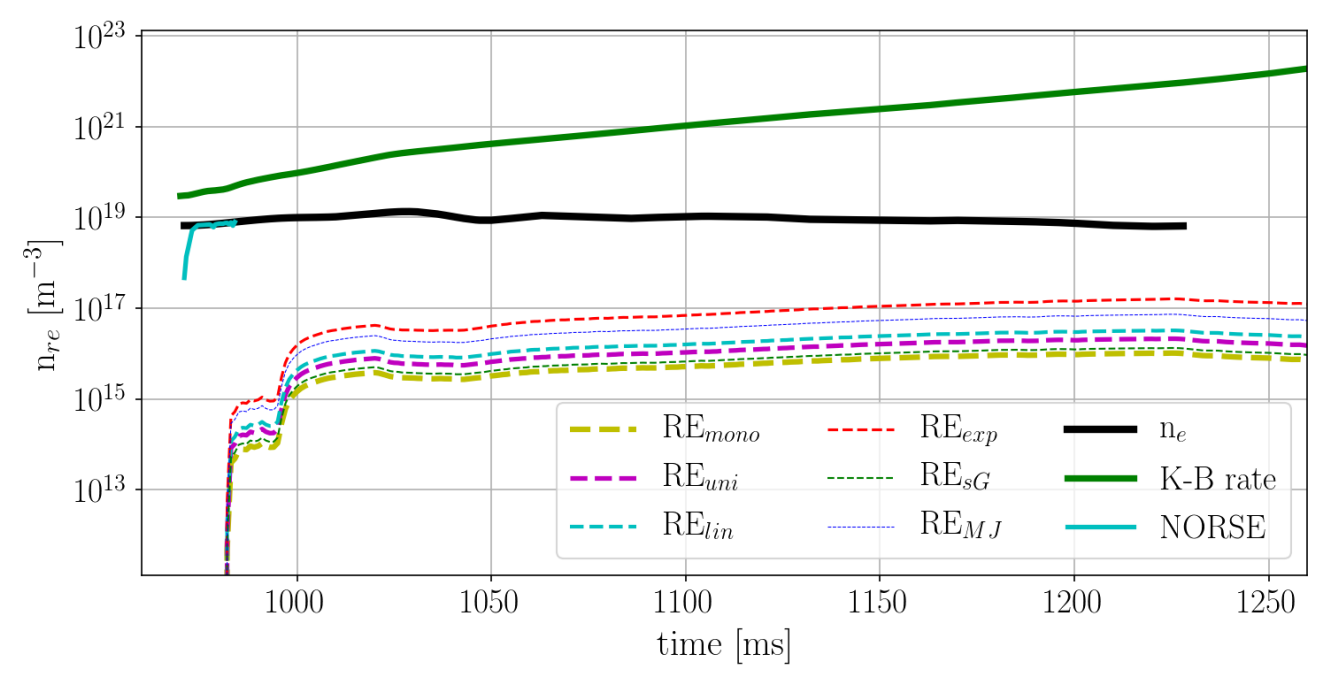

Figure 3. Time traces of total electron density (black) measured with interferometer, RE density from Kruskal-Bernstein theory (green), RE density from NORSE simulation (cyan) and RE densities corresponding to RE currents from Figure 2c (dashed lines). 


\section{Application: RE Influence on Ramp-Up}

REs are frequently generated during the current ramp-up phase in the COMPASS tokamak. Here, the influence of the RE generation on the plasma current $I_{p}$ at the end of the ramp-up phase is investigated using the reported model for the RE current $I_{R E}$.

The density scan in the range $1-5 \times 10^{19} \mathrm{~m}^{-3}$ was done during the second RE campaign. The scan consisted of 10 COMPASS discharges from \#8552 to \#8561. Plasma current $I_{p}$ was feedback controlled to $130 \mathrm{kA}$ during the flat-top phase and the toroidal magnetic field $B_{\text {tor }}$ was $1.15 \mathrm{~T}$ for all discharges.

Figure 4 a presents an estimate of $n_{R E}$, which is more suitable for comparison of the method reported here with Kruskal-Bernstein and NORSE theories. The three discharges were chosen to cover the standard tokamak discharge (\#8553), the slide-away regime (\#8559) and the transient between the previous two (\#8555). To avoid redundant lines, $n_{R E}$ was calculated only for the two most extreme REDF- the monoenergetic $f_{\text {mono }}$ and the exponential $f_{\text {exp }}$ ones.

The discharge $\# 8553$ has $n_{R E}$ lower by an order of magnitude during the ramp-up phase than the other two discharges (see Figure 4). Surprisingly, discharges \#8555 and \#8559 have similar $n_{R E}$ at the beginning of the discharge. However, $n_{R E}$ drops for the former discharge as expected from the missing $\beta_{p}$ rise from EFIT and density drop, as observed by the Thomson Scattering system. Finally, the corresponding RE current $I_{R E}$ at the end of the ramp-up phase for discharges \#8553, \#8555 and \#8559 are 0.3-2.0 kA, 2.3-17.8 kA and 2.4-19.6 kA, respectively. Therefore, the expected trend is observed. Note that the non-continuous line for discharge \#8553 comes from the defined thresholds of the method.
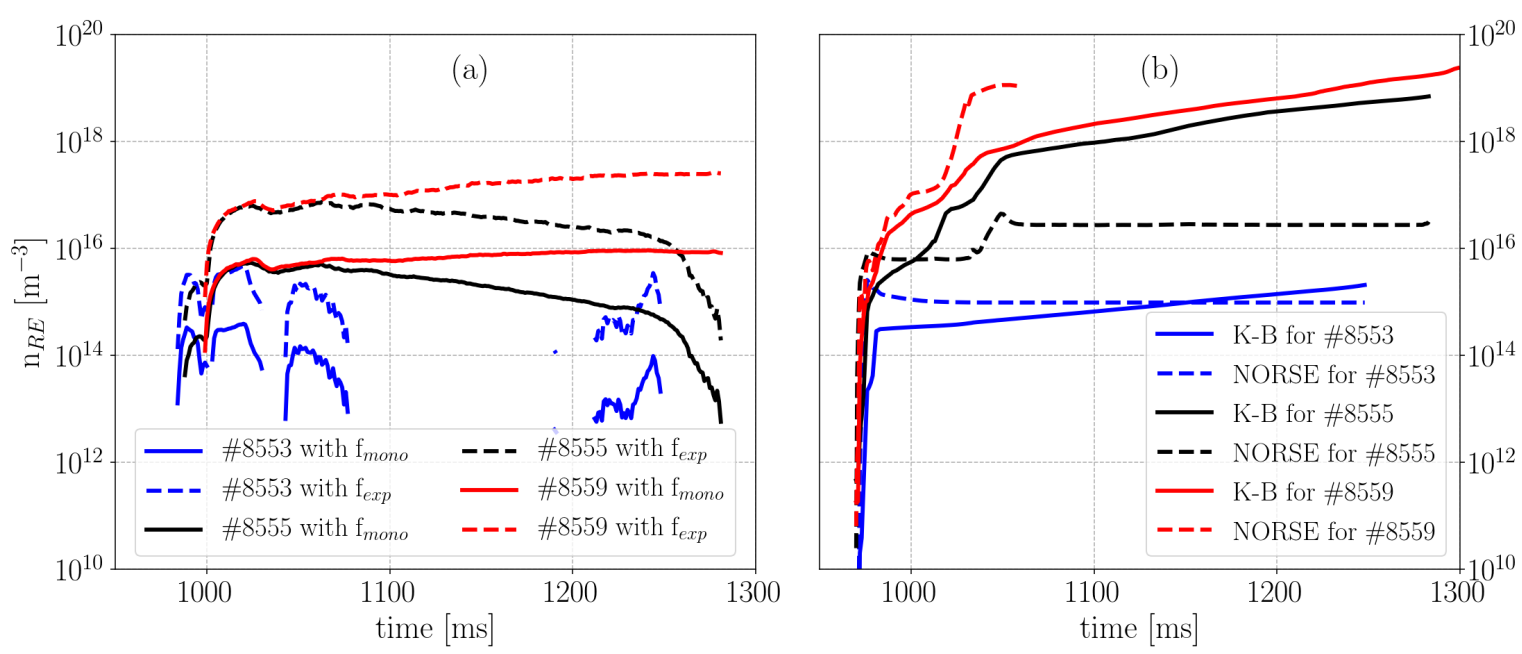

Figure 4. Time traces of the estimated $n_{R E}$ for the three typical discharges: \#8553 (blue, standard discharge), \#8555 (black, intermediate case) and \#8559 (red, slide-away regime). (a) Estimates from measurements where for each discharge the two most extreme REDF: the monoenergetic $f_{\text {mono }}$ (full line) and the exponential $f_{\exp }$ (dashed line). (b) Estimates from Kruskal-Bernstein theory (solid line) and NORSE simulation (dashed line).

To complete the analyses, Kruskal-Bernstein theory and NORSE code were used to theoretically estimate $n_{R E}$. The results are presented in Figure $4 \mathrm{~b}$.

First to notice from Figure 4 is that both theories have the expected trend in density of RE rising from \#8553 to \#8559. Differently from discharge \#7298 in Figure 3, the Kruskal-Bernstein theory does not give $n_{R E}$ over $n_{e}$, while $n_{R E}$ do rise towards $n_{e}$ at the end of discharge for \#8555 and \#8559. For these two discharges, the Kruskal-Bernstein theory does not show a significant difference in $n_{R E}$. On the other hand, NORSE estimates that those two discharges are quite different-according to this code \#8559 reaches slide-away regime already around $1060 \mathrm{~ms}$, which is probably too early.

Interestingly, in the case of standard discharge \#8553, both codes seem to approximately agree in the order of magnitude with the experimental $n_{R E}$ (between $10^{14}$ and $10^{15} \mathrm{~m}^{-3}$ ). The same is 
valid when the NORSE simulation is compared with experimental $n_{R E}$ using $f_{\text {exp }}$ for discharge \#8555. However, the NORSE code is not made to predict the drop of $n_{R E}$, clearly observed in Figure 4a and from $\beta_{\text {pol }}$ from the EFIT reconstruction.

\section{Application: RE Localisation}

In this section, we investigate the RE energy limit from the RE outward drift through the analytically derived calculation reported by Zehrfeld [18].

Zehrfeld's analysis provides the $W_{\text {drift }}$ as a function of the normalized minor radius $\rho=r / a_{p}$. One of the main messages from this analysis is that $W_{\text {drift }}$ profile can have (depending on machine and plasma parameters) maximum value inside plasma (i.e., $\rho_{\max }<1$-dashed coinciding colored lines in Figure 5), which indicates that REs of certain energy can loose confinement before they reach the plasma edge (i.e., the last closed flux surface) that is represented by $R_{\text {out }}$ in Figure 5. Namely, peaking parameter of the plasma current profile $m^{1}$ proved to be the most significant parameter for RE confinement in respect to the drift. Therefore, knowledge of the $W_{\text {drift }}(\rho)$ profile can be used for localization of the RE beam. Knowing $W_{\max }$ at a given time of the discharge, one can find the minimum minor radius $\rho_{\min }$ (solid colored lines in Figure 5) where $W_{\text {drift }}\left(\rho_{\min }\right)=W_{\max }$, corresponding to the minor radius below which REs with energy $W_{\max }$ cannot be confined.
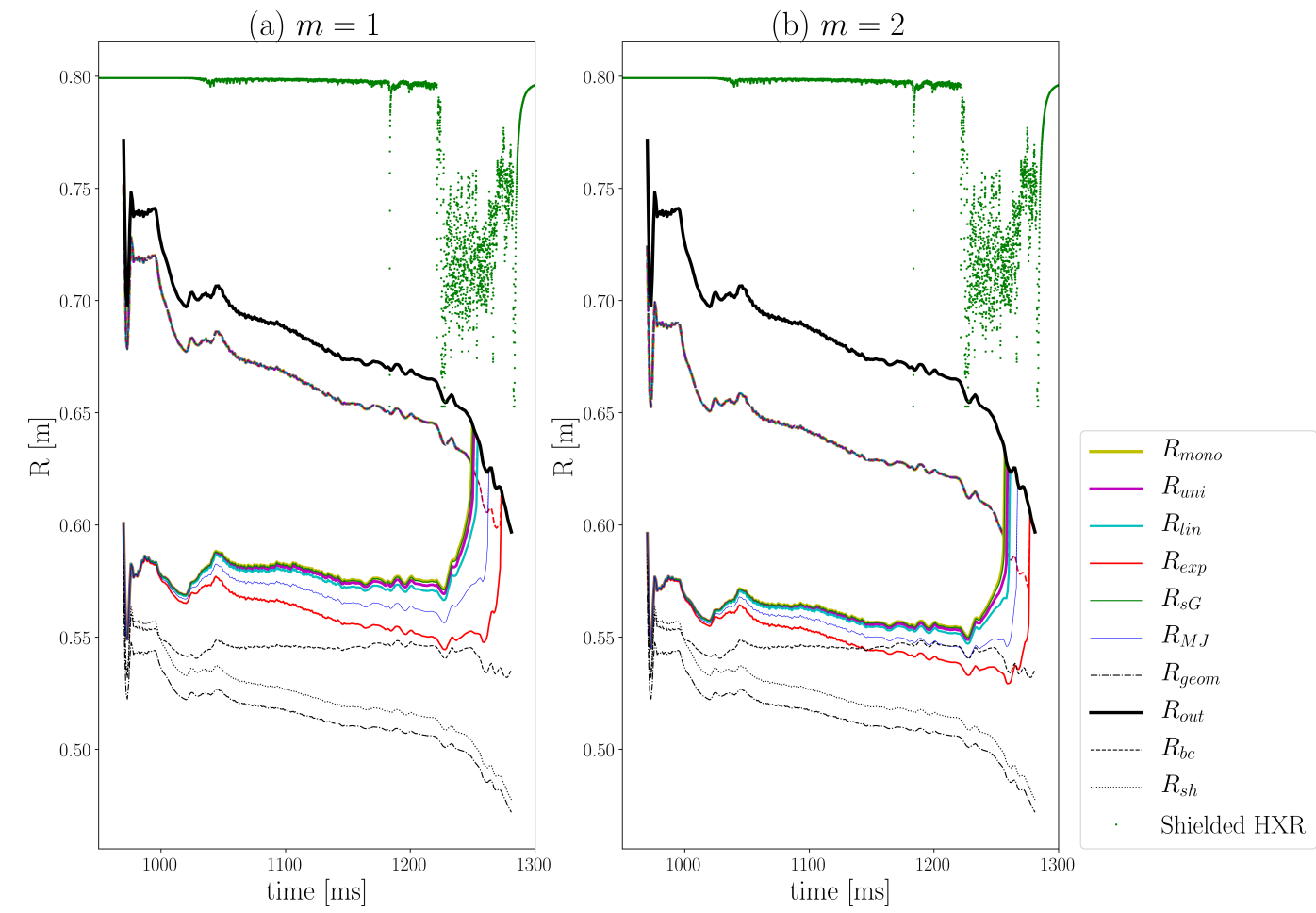

Figure 5. Time traces for lower $\rho_{\min }$ (solid colored lines) and upper $\rho_{\max }$ (dashed coinciding colored lines) limits of RE major radii for all six REDFs are presented for discharge \#7298. For orientation, major radius of plasma geometrical center $R_{\text {geom }}$ (black dash-dotted line), outer plasma major radius $R_{\text {out }}$ (black solid line), plasma current barycenter $R_{b c}$ (black dashed line) and theoretical bulk plasma barycenter from Shafranov shift $R_{s h}$ (black dotted line) are added. Beside all major radii, the Shielded HXR signal (green points) is presented. Figure is plotted for two of current profile factor $m$ values: (a) $m=1$; and (b) $m=2$.

$1 \quad m$ is plasma current profile $I(\rho)$ peaking factor, defined as: $I(\rho)=I_{p}\left(1-\left(1-\rho^{2}\right)^{m+1}\right)$. 
Generally, the major radius of plasma geometrical center $R_{\text {geom }}$ and bulk plasma barycenter $R_{s h}$ do not coincide in the tokamak plasma due to the Shafranov shift [19]. Additionally, there is RE beam present in the observed discharges shifting the plasma current barycenter $R_{b c}$ more outwards. All these radii $R_{\text {geom }}, R_{s h}$ and $R_{b c}$ are taken from EFIT and shown in Figure 5 for orientation purposes.

EFIT predicts $m=1.5$ while Zehrfeld's method assumes $m$ to be an integer. Accordingly, we used both rounding integers values: $m=1$ and $m=2$.

One can notice from Figure 5 that RE orbits are predicted in minor radius of $10-15 \mathrm{~cm}$, which is not very limiting knowing that the COMPASS minor plasma radius is around $20 \mathrm{~cm}$. RE orbits are localized more inside the plasma for higher $m$, as could be expected due to the higher peaking of $W_{\text {drift }}$ profile. For the same reason, later significant losses of high energy RE are expected for higher $m$, which can be seen by slightly delayed equalization of the RE radius with $R_{\text {out }}$ (for 5-15 ms) for factor of current profile $m=2$ than in the $m=1$ case. However, both timings for significant high energy RE losses are a few tens of milliseconds after the Shielded HXR observes the losses, showing that these losses are probably due to the RE outward drift as $I_{p}$ is decreasing and connection of strong Shielded HXR signal and $I_{p}$ ramp-down phase is indeed observed in Figure $2 \mathrm{~b}$. On the other hand, this does not explain the interaction on the high-field side seen by the visible cameras.

\section{Conclusions}

Understanding RE physics gives us better prediction towards understanding RE generation and mitigation in ITER. One basic RE parameter to compare model and experiment is the RE current. Therefore, we have presented here one method for estimating the RE current in the COMPASS tokamak.

The pitch angle showed to be relevant for the RE energy calculation, which plays an internal role in the RE current estimate method. Implementation of the method on the COMPASS discharge \#7298 shows that at least a few kA of the total plasma current is driven by the RE. The method is then used for $n_{e}$-scan and showed the different amount of RE current present in the plasma at the expected trend. Namely, discharges with lower density have higher RE current. Commonly used Kruskal-Bernstein theory does not appear suitable for high RE current discharges. NORSE, a code built for such discharges, showed to be more sensitive to plasma parameters, but further development is necessary for better prediction of the RE dynamics. Applying the knowledge of RE current to the RE drift energy limit, a crucial role of the limit at the final stage of the COMPASS plasma discharge, is reported.

At the first instance, an implementation of the RE current into the equilibrium simulation is left for future work on the COMPASS tokamak. Furthermore, theories used here are also the most commonly used model basis in the RE research community. However, they obviously overestimate the number of REs. Their further development is far beyond the scope of this article. The results obtained from theories are here to show the issue is oversimplified and demonstrate the need for a better modeling of the RE generation.

Author Contributions: Conceptualization, M.V.; methodology, M.V.; software, M.V., O.F., E.M. and COMPASS IT Team; validation, M.V.; formal analysis, M.V.; investigation, M.V., O.F. and J.M.; resources, J.M.; data curation, M.V. and J.M.; writing-original draft preparation, M.V.; writing-review and editing, M.V., O.F., J.M, and E.M; visualization, M.V. and O.F.; supervision, J.M.; project administration, J.M. and COMPASS department; and funding acquisition, J.M., EUROfusion and COMPASS department.

Funding: This research was funded by Ministry of Education, Youth and Sport of the Czech Republic (MEYS) grant number LM2011021 that supported COMPASS operation and by the Euratom research and training programme 2014-2018 under grant agreement No 633053 with the Co-fund by MEYS project number 8D15001.

Acknowledgments: Erasmum Mundus Joint Doctoral Collage in Fusion Science and Engineering (often abbreviated as "Fusion-DC") is acknowledged for providing the necessary infrastructure to the first author for his studies. Moreover, we would like to thank to the whole COMPASS team of scientist, engineers and technicians for their fruitful collaboration.

Conflicts of Interest: The founding sponsors had no role in the design of the study; in the collection, analyses, or interpretation of data; in the writing of the manuscript, or in the decision to publish the results. In addition, the views and opinions expressed in this work do not necessarily reflect those of the European Commission. 


\section{Abbreviations}

The following abbreviations are used in this manuscript:

RE Runaway Electron

REDF Runaway Electron Distribution Function

HXR Hard X-ray

\section{References}

1. Hender, T.; Wesley, J.; Bialek, J.; Bondeson, A.; Boozer, A.; Buttery, R.; Garofalo, A.; Goodman, T.; Granetz, R.; Gribov, Y.; et al. Chapter 3: MHD stability, operational limits and disruptions. Nucl. Fusion 2007, 47, S128. [CrossRef]

2. Pánek, R.; Adámek, J.; Aftanas, M.; Bílková, P.; Böhm, P.; Cahyna, P.; Cavalier, J.; Dejarnac, R.; Dimitrova, M.; Grover, O.; et al. Status of the COMPASS tokamak and characterization of the first H-mode. Plasma Phys. Control. Fusion 2016, 58, 014015. [CrossRef]

3. Fujita, T.; Fuke, Y.; Yoshida, Z.; Inoue, N.; Tanihara, T.; Mori, K.; Fukao, M.; Tomita, Y.; Mohri, A. High-Current Runaway Electron Beam in a Tokamak Plasma. J. Phys. Soc. Jpn. 1991, 60, 1237-1246. [CrossRef]

4. Havlíček, J.; Hronová, O. Magnetic Diagnostics of COMPASS Tokamak. 2010. Available online: http:/ / www.ipp.cas.cz/vedecka_struktura_ufp/tokamak/tokamak_compass/diagnostics/magnetickadiagnostika/index.html (accessed on 16 January 2019).

5. Bilkova, P.; Aftanas, M.; Bohm, P.; Weinzettl, V.; Sestak, D.; Melich, R.; Stockel, J.; Scannell, R.; Walsh, M. Design of new Thomson scattering diagnostic system on COMPASS tokamak. Nucl. Instrum. Methods Phys. Res. Sect. A 2010, 623, 656-659. [CrossRef]

6. Mlynar, J.; Ficker, O.; Vlainic, M.; Weinzettl, V.; Imrisek, M.; Paprok, R.; Rabinski, M.; Jakubowski, M.; the COMPASS Team. Effects of Plasma Control on Runaway Electrons in COMPASS Tokamak. In Proceedings of the 42nd EPS Conference on Plasma Physics, Lisbon, Portugal, 22-26 June 2015; Number P4.102 in ECA; Volume 39E.

7. Ficker, O.; Mlynar, J.; Vlainic, M.; Macusova, E.; Vondracek, P.; Weinzettl, V.; Urban, J.; Cerovsky, J.; Cavalier, J.; Havlicek, J.; et al. Long slide-away discharges in the COMPASS tokamak. In Proceedings of the 58th Annual Meeting of the APS Division of Plasma Physics, San Jose, CA, USA, 31 October-4 November 2016; Volume 61.

8. Artaud, J.; Imbeaux, F.; Garcia, J.; Giruzzi, G.; Aniel, T.; Basiuk, V.; BÃcoulet, A.; Bourdelle, C.; Buravand, Y.; Decker, J.; et al. Metis: a fast integrated tokamak modelling tool for scenario design. Nucl. Fusion 2018, 58, 105001. [CrossRef]

9. Vlainić, M.; Mlynář, J.; Weinzettl, V.; Papřok, R.; Imrísek, M.; Ficker, O.; Vondráček, P.; Havlíček, J. First Dedicated Observations of Runaway Electrons in COMPASS Tokamak. Nukleonika 2015, 60, 249-255. [CrossRef]

10. Vlainic, M. Study of Runaway Electrons at COMPASS Tokamak. Ph.D. Thesis, Ghent University, Ghent, Belgium, 2017.

11. Yu, J.H.; Hollmann, E.M.; Commaux, N.; Eidietis, N.W.; Humphreys, D.A.; James, A.N.; Jernigan, T.C.; Moyer, R.A. Visible imaging and spectroscopy of disruption runaway electrons in DIII-D. Phys. Plasmas 2013, 20, 042113. [CrossRef]

12. Pankratov, I.M. Analysis of the synchrotron radiation spectra of runaway electrons. Plasma Phys. Rep. 1999, 25, 145-148.

13. Azzalini, A. The Skew-Normal and Related Families; Institute of Mathematical Statistics Monographs, Cambridge University Press: Cambridge, UK, 2013.

14. Jüttner, F. Das Maxwellsche Gesetz der Geschwindigkeitsverteilung in der Relativtheorie. Ann. Phys. 1911, 339, 856-882. [CrossRef]

15. Vlainic, M.; Vondracek, P.; Mlynar, J.; Weinzettl, V.; Ficker, O.; Varavin, M.; Paprok, R.; Imrisek, M.; Havlicek, J.; Panek, R.; et al. Synchrotron Radiation from Runaway Electrons in COMPASS Tokamak. In Proceedings of the 42nd EPS Conference on Plasma Physics, Lisbon, Portugal, 22-26 June 2015; Number P4.108 in ECA; Volume 39E. 
16. Connor, J.; Hastie, R. Relativistic limitations on runaway electrons. Nucl. Fusion 1975, 15, 415. [CrossRef]

17. Stahl, A.; Landreman, M.; Embréus, O.; Fülöp, T. NORSE: A solver for the relativistic non-linear Fokker-Planck equation for electrons in a homogeneous plasma. Comput. Phys. Commun. 2017, 212, 269-279. [CrossRef]

18. Zehrfeld, H.P.; Fussmann, G.; Green, B.J. Electric field effects on relativistic charged particle motion in Tokamaks. Plasma Phys. 1981, 23, 473. [CrossRef]

19. Shafranov, V.D. Equilibrium of a toroidal pinch in a magnetic field. Soviet Atomic Energy 1962, 13, 1149-1158. [CrossRef]

(C) 2019 by the authors. Licensee MDPI, Basel, Switzerland. This article is an open access article distributed under the terms and conditions of the Creative Commons Attribution (CC BY) license (http:/ / creativecommons.org/licenses/by/4.0/). 